\title{
Aspectos Farmacoeconômicos do Tratamento da Esquizofrenia no âmbito da Assistência Farmacêutica Especializada
}

\author{
Emanuela Machado Silva Saraiva' ${ }^{1}$ Julyane Taisa Barros ${ }^{2}$
}

\begin{abstract}
Resumo: O Componente Especializado é um programa de acesso a medicamentos na esfera do Sistema Único de Saúde que garante o tratamento medicamentoso através dos Protocolos Clínicos e Diretrizes Terapêuticas, destinados à várias doenças entre elas a esquizofrenia que é uma doença crônica, caracterizada por um transtorno mental, que pode atingir ambos os sexos de idades variáveis, com causa desconhecida. $\mathrm{O}$ presente estudo possui natureza descritiva, exploratória, retrospectiva, com abordagem quantitativa. Realizado no Componente Especializado da Assistência Farmacêutica no município de Missão Velha - Ceará por meio de um levantamento de dados referentes a repasses financeiros mensais relativos aos medicamentos antipsicóticos destinados aos pacientes com esquizofrenia cadastrados no referido serviço de saúde, durante o ano de 2015. Os resultados apontaram que o medicamento utilizado no tratamento da esquizofrenia mais oneroso foi a Quetiapina $200 \mathrm{mg}$ que totalizou $\mathrm{R} \$ 21.363,98$, seguida da Olanzapina $10 \mathrm{mg}$ e $5 \mathrm{mg}$ que obtiveram um total de $\mathrm{R} \$ 13.253,40$ e $\mathrm{R} \$ 11.754,61$, respectivamente. A Risperidona $2 \mathrm{mg}$ foi o medicamento que menos onerou o montante financeiro, a qual totalizou $\mathrm{R} \$ 210,00$ no período considerado pela pesquisa. O custo anual total foi de $\mathrm{R} \$ 55.867,70$ com medicamentos antipsicóticos destinados a atender a demanda de 62 pacientes cadastrados no serviço de saúde. O Componente Especializado da Assistência Farmacêutica é importante pois, garante o acesso do cidadão ao tratamento seguro e eficaz, bem como reduz os custos para o município e para o paciente.
\end{abstract}

Palavras-Chaves: Assistência Farmacêutica. Esquizofrenia. Farmacoeconomia. Protocolos.

\section{Pharmacoeconomic Aspects of the Treatment of Schizophrenia in the scope of Specialized Pharmaceutical Assistance}

\begin{abstract}
The Specialized Component is a program of access to medicines in the sphere of the Unified Health System that guarantees the medical treatment through the Clinical Protocols and Therapeutic Guidelines, destined to several diseases among them the schizophrenia that is a chronic disease, characterized by a mental disorder, which can reach both sexes of varying ages, with unknown cause. The present study has a descriptive, exploratory, retrospective nature, with a quantitative approach. Made in the Specialized Component of Pharmaceutical Assistance in the municipality of Missão Velha - Ceará by means of a survey of data referring to monthly financial transfers related to antipsychotic drugs for patients with schizophrenia registered in said health service during the year 2015. The results showed that the drug used to treat

\footnotetext{
${ }^{1}$ Bacharel em FARMÁCIA pela Universidade de Fortaleza - UNIFOR (2007), com especialização em Gestão de Varejo pela Faculdades Nordeste - FANOR (2011). Em fase de conclusão do curso de pós-graduação em Docência do Ensino Superior, pela Faculdade de Juazeiro do Norte-FJN. Mestranda em Ciências da Saúde pela Faculdade de Medicina do ABC - FMABC desenvolve pesquisa com foco em cuidados farmacêuticos na atenção básica de saúde. Com experiência em farmácia comercial, gestão em saúde e docência em saúde. Atualmente seu exercício profissional engloba as áreas de assistência e atenção farmacêutica, gestão em serviço público de saúde e docência no curso de graduação em Farmácia na cidade de Juazeiro do Norte-CE; Contato: emanuelams@ @otmail.com;

${ }^{2}$ Bacharel em FARMÁCIA pela Faculdade de Juazeiro do Norte - FJN. Contato: jubarros26@ gmail.com
} 
schizophrenia most costly was Quetiapine $200 \mathrm{mg}$, which totaled R \$21,363.98, followed by Olanzapine $10 \mathrm{mg}$ and $5 \mathrm{mg}$, which obtained a total of $\mathrm{R} \$ 13,253.40$ and $\mathrm{R} \$ 11,754.61$, respectively. Risperidone $2 \mathrm{mg}$ was the medicine that less burdened the financial amount, which totaled $\mathrm{R} \$ 210,00$ in the period considered by the research. The total annual cost was $\mathrm{R} \$ 55,867.70$ with antipsychotic drugs intended to meet the demand of 62 patients enrolled in the health service. The Specialized Component of Pharmaceutical Care is important because it guarantees the citizen's access to safe and effective treatment, as well as reduces costs for the municipality and the patient.

Keywords: Schizophrenia. Specialized Component. Ecominics, Pharmacetical. Protocols.

\section{Introdução}

A esquizofrenia é uma das mais graves doenças neuropsiquiátricas, que atinge cerca de $1 \%$ da população mundial. No Brasil a esquizofrenia ocupa $30 \%$ dos leitos psiquiátricos hospitalares (PULL, 2005).

Os sinais e sintomas iniciais surgem na adolescência ou no início da vida adulta e apresentam-se por sintomas psicóticos como delírios e alucinações, discurso e comportamento sem organização ou catiônicos, e restrições da expressividade emocional caracterizada como um sintoma negativo (EPAMINONDAS, 2010).

Segundo Gelder, Mayou e Cowen (2006), a doença ocorre entre 15 e 45 anos, sendo o início nos homens é cerca de 5 anos mais baixa que nas mulheres. Aqueles apresentam um risco 1,4 a 2,3 vezes maiores do que as mulheres de desenvolver esquizofrenia, sendo o pico de incidência aos 15 aos 25 anos nos homens e 25 a 35 anos nas mulheres (DA SILVA, 2006).

Apresenta curso variável, em que aproximadamente 30\% dos casos apresentam recuperação completa ou quase completa, $30 \%$ com remissão incompleta e prejuízo parcial de funcionamento $30 \%$ com deterioração importante e persistente da capacidade de funcionamento profissional, social e afetivo (BRASIL $\left.{ }^{\mathrm{a}}, 2013\right)$.

De acordo com o Protocolo Clínico e Diretrizes Terapêuticas (PCDT) para esquizofrenia, o diagnóstico é clínico e baseia- se nos critérios da CID-10 que disserta sobre os aspectos gerais que precisam ser atendidos para o usuário se encaixar nas exigências do serviço de saúde (BRASIL ${ }^{\mathrm{a}}$, 2013). 
O Ministério da Saúde preconiza que o tratamento medicamentoso é feito com antipsicóticos analisados por meio de estudos, que fundamentam a recomendação do protocolo, de maneira que os fármacos destinados ao tratamento da esquizofrenia são: Risperidona, Quetiapina, Ziprasidona, Olanzapina, Clozapina disponibilizados através do Componente Especializado da Assistência Farmacêutica e os medicamentos: Clorpromazina, Haloperidol e Decanoato de Haloperidol disponíveis através do Componente Básico da Assistência Farmacêutica (BRASIL ${ }^{\mathrm{a}}$, 2013).

Exceto a clozapina, todos os antipsicóticos, podem ser usados no tratamento, sem ordem de preferência, dos usuários com diagnóstico de esquizofrenia que preencham os critérios de inclusão. Os tratamentos devem ser feitos com um medicamento de cada vez, baseado no perfil de segurança e na tolerabilidade do paciente. Em caso de falha terapêutica que se caracteriza como o uso de qualquer desses fármacos pelo menos 6 semanas, nas doses adequadas, sem melhora de no mínimo de 30\% na escala de Avaliação Psiquiátrica Breve - BPRS, deverá ser feita uma segunda tentativa com algum outro antipsicótico (BRASIL ${ }^{\mathrm{a}}$, 2013).

O Componente Especializado da Assistência Farmacêutica - CEAF é uma estratégia de acesso a medicamentos na esfera do Sistema único de Saúde - SUS, que procura a garantia da plenitude do tratamento medicamentoso no ambulatório em que as linhas de cuidado são definidas em Protocolos Clínicos e Diretrizes Terapêuticas publicadas pelo Ministério da Saúde (BRASIL $\left.{ }^{\mathrm{a}}, 2013\right)$.

Diante disso, o objetivo da presente pesquisa foi avaliar os custos com medicamentos antipsicóticos destinados ao tratamento da esquizofrenia no âmbito do Componente Especializado da Assistência Farmacêutica do município de Barbalha-Ceará durante o ano de 2015.

\section{Metodologia}

O presente estudo é de natureza descritiva, exploratória, retrospectiva, com abordagem quantitativa, tendo sido realizado no Componente Especializado da Assistência Farmacêutica do município de Missão Velha - CE. 
A cidade de Missão Velha - CE localiza-se ao sul do estado do Ceará e compõe a região metropolitana do Cariri. Dados do Instituto Brasileiro de Geografia e Estatística - IBGE, estimam que a cidade possuía em 2017 aproximadamente 35.409 habitantes, caracterizando-se como um município de pequeno porte.

O Componente Especializado da Assistência Farmacêutica do município faz parte das unidades e serviços de saúde pública disponibilizados à população.

$\mathrm{Na}$ presente pesquisa, foram considerados os repasses financeiros referentes apenas aos medicamentos antipsicóticos destinados ao cadastro de usuários portadores de esquizofrenia atendidos pelo serviço supracitado, referente ao ano de 2015.

Foi realizado um estudo de consumo médio mensal por meio de um levantamento de dados referentes a repasses financeiros de medicamentos antipsicóticos através de notas fiscais emitidas por relatórios gerados no sistema HÓRUS.

A análise de dados foi realizada através de estatística para cálculo de média e desvio padrão, os quais subsidiaram a elaboração de gráficos e tabelas.

A pesquisa atendeu aos preceitos éticos da Resolução no 466 de dezembro de 2012, do Conselho Nacional de Saúde, tendo em vista a garantia do anonimato e a guarda do sigilo das informações relativas aos seres humanos (BRASIL, 2012).

\section{Resultados e Discussão}

De acordo com os dados obtidos mediante a avaliação dos registros do Componente Especializado da Assistência Farmacêutica da cidade de Missão Velha-CE, obteve-se o gasto anual referente ao fornecimento de medicamentos para o tratamento de 62 pacientes com esquizofrenia. 
Gráfico 1. Distribuição dos gastos conforme o medicamento destinado ao tratamento da esquizofrenia.

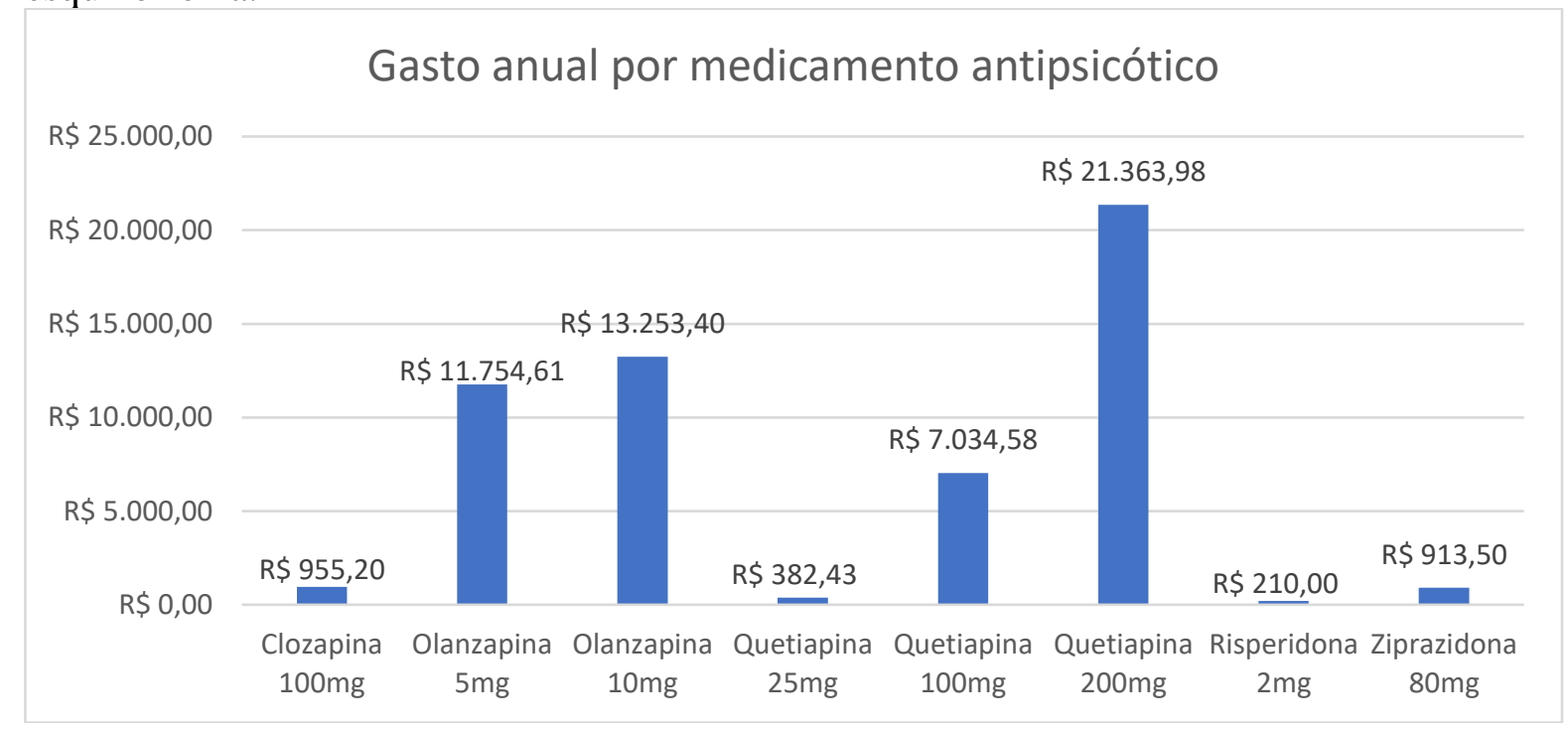

Fonte: Dados da pesquisa, 2016.

Conforme os dados expressos no gráfico 1, observa-se que o medicamento utilizado no tratamento da esquizofrenia mais oneroso foi a Quetiapina 200mg, que totalizou $\mathrm{R} \$ 21.363,98$, seguida da Olanzapina 10mg e 5mg que obtiveram um total de $\mathrm{R} \$ 13.253,40$ e $\mathrm{R} \$ 11.754,61$, respectivamente. A Risperidona $2 \mathrm{mg}$ foi o medicamento que menos onerou o montante financeiro, a qual totalizou $\mathrm{R} \$ 210,00$ no período considerado pela pesquisa.

O fato da Risperidona ter tido pouco repasse pode ser justificada por critérios estabelecidos pelo PCDT (2013), que indicam que para pacientes com insuficiência renal ou hepática a dose máxima de administração de $3 \mathrm{mg} /$ dia não deve ser utilizada, fazendo com que a risperidona não seja a primeira alternativa no tratamento da esquizofrenia.

No entanto os gastos com a Olanzapina $5 \mathrm{mg}$ e $10 \mathrm{mg}$ poderiam ser maiores, tendo em vista que durante os meses de janeiro e março não houve envio desses medicamentos pela esfera governamental.

O medicamento a base de Quetiapina 25mg, também não obteve repasse nos meses de janeiro, fevereiro, julho, agosto, setembro, outubro e novembro. A ausência de repasse também ocorreu para Clozapina $100 \mathrm{mg}$, nos meses de janeiro, março, julho e agosto não houve repasse do medicamento. 
Segundo a Portaria ${ }^{\circ} 1.554$, de 30 de julho de 2013 que dispõe sobre as regras de financiamento e execução do CEAF no âmbito do Sistema Único de Saúde (SUS), Componente Especializado é uma técnica de acesso a medicamentos na esfera do SUS, que será assegurado mediante a pactuação entre a União, Estados, Distrito Federal e Municípios (BRASIL ${ }^{\mathrm{b}}$,2013).

A Ziprasidona 80mg não teve seu repasse mensal interrompido durante o ano de $2015 \mathrm{e}$ esse medicamento é recomendado em caso de intolerância a clozapina por agranulocitose, após sua indicação por refratariedade. Segundo o PCDT (2013) mulheres em idade fértil devem ser esclarecidas quanto à necessidade do uso regular de métodos contraceptivos e, em caso de dúvida, sugere-se teste de gravidez antes do início do tratamento. Também não se recomenda o uso de ziprasidona durante a lactação (BRASIL ${ }^{\mathrm{a}}$, 2013).

\section{Considerações Finais}

Em se tratando de um município de pequeno porte, a cidade de Missão Velha - CE apresentou um montante financeiro no valor de $\mathrm{R} \$ 55.867,70$, no ano de 2015 , referente ao fornecimento de medicamentos utilizados no tratamento de esquizofrenia.

$\mathrm{O}$ referido recurso, demonstra que gastos destinados à disponibilização de medicamentos à população são necessários e como estes são disponibilizados pelo Componente Especializado da Assistência Farmacêutica deixam de onerar os recursos públicos do município, já que são de responsabilidade das esferas estadual e federal.

Do ponto de vista do paciente, o Componente Especializado da Assistência Farmacêutica é importante pois, garante o acesso ao tratamento medicamentoso de maneira gratuita e, consequentemente, atua na redução custos para o mesmo.

\section{Referências}

BRASIL. Resolução $n^{\circ} 466$ de dezembro de 2012: Aprovar as seguintes diretrizes e normas regulamentadoras de pesquisas envolvendo seres humanos. Brasília - DF, 2012. 
BRASIL a Portaria SAS/MS N ${ }^{o}$ 364, de 9 de abril de 2013. Aprova o Protocolo Clínico e Diretrizes Terapêuticas - Esquizofrenia. Brasília - DF, 2013.

BRASIL ${ }^{\mathrm{b}}$. Portaria $N^{o}$ 1554, de 30 de julho de 2013: Dispõe sobre as regras de financiamento e execução do Componente Especializado da Assistência Farmacêutica no âmbito do Sistema Único de Saúde (SUS). Brasília - DF, 2013.

DA SILVA, R.C.B. Esquizofrenia: uma revisão. Psicologia USP, v. 17, n. 4, p. 263-285, 2006.

EPAMINONDAS, F.R. Modelagem do Comportamento para Controle da Esquizofrenia. Goiânia, 2010.

GELDER, M.; MAYOU, R.; COWEN, P. Tratado de Psiquiatria. Rio de Janeiro: Guanabara Koogan,2006.

PULL, C. Diagnóstico da esquizofrenia: uma revisão. In M. Maj \& N. Sartorius (Orgs.), Esquizofrenia (pp. 13-70). Porto Alegre: Artmed.

SARAIVA, Emanuela Machado Silva; BARROS, Julyane Taisa. Economic Evaluation of the Treatment of Schizophrenia in the Specialized Composition of Pharmaceutical Assistance in the Municipality of Missão Velha-CE.

\section{Como citar este artigo (Formato ABNT):}

SARAIVA, Emanuela Machado Silva; BARROS, Julyane Taisa. Aspectos Farmacoeconômicos do Tratamento da Esquizofrenia no âmbito da Assistência Farmacêutica Especializada. Id on Line Rev.Mult. Psic., 2018, vol.12, n.42, p. 634-640. ISSN: 1981-1179.

Recebido: 08/10/2018;

Aceito: 11/10/2018 